Dix-neuvièmes journées nationales de la Fédération française d'étude de la reproduction

(Issy-les-Moulineaux, 17-19 septembre 2014)

\title{
Le donneur dans les récits de conception : pratiques narratives de parents ayant recours au don de sperme
}

\section{Donor in conception stories: Narrative practices by parents conceiving with donated spermatozoa}

\author{
M. Doumergue*, N. Kalampalikis \\ Laboratoire GRePS (EA 4163), université Lyon 2, 5, avenue P. Mendès-France, 69500 Bron, France
}

\section{N F O A R T I C L E}

\section{Historique de l'article :}

Reçu le 4 juin 2014

Accepté le 15 juillet 2014

Disponible sur Internet le 19 août 2014

\section{Mots clés :}

Récits de conception

Parenté

Don de spermatozoïdes

\begin{abstract}
R É S U M É
La population des parents d'enfants nés par procréation avec donneur est peu étudiée dans le contexte français, où le donneur est anonyme. La présente recherche associe une opération quantitative appréhendant les attitudes et décisions des parents quant au récit de conception à l'enfant, et un volet qualitatif visant également, plus largement, leur vécu de ce mode de parenté. Les résultats issus de données systématiques sur le devenir des récits de conception ( $n=929$ requérants, dont 216 parents) confirment une décision majoritaire des parents de parler à l'enfant de leur recours au don. L'analyse des entretiens semi-directifs réalisés auprès de 37 parents souligne un paradoxe entre la présence d'un donneur dans les récits, objectivé en tant que graine, et un projet représentationnel qui s'ancre dans des modes de parenté normalisés.
\end{abstract}

(c) 2014 Elsevier Masson SAS. Tous droits réservés.

\section{Keywords:}

Conception stories

Kinship

Spermatozoa donation

\section{A B S T R A C T}

Little is known about the parents of donor-conceived offspring in France, where the anonymity of the donor prevails. The present study associates quantitative data informing the attitudes and decisions of parents towards disclosure and qualitative data related to their experience of being parents of a donorconceived offspring. The quantitative results about the becoming of the conception narratives $(n=929$ requesting people, including 216 parents) confirmed that most parents decided to tell their child about the donor conception. The analysis of the semi-structured interviews of 37 parents defined the existence of a paradox between the conception stories mentioning the donor, who is objectified as a seed, and the representational project parents mostly pursue, which is anchored in a normalisation process.

(c) 2014 Elsevier Masson SAS. All rights reserved.

\section{Introduction}

En France, l'anonymat du donneur est un choix législatif reconduit dans un paysage international mouvant ${ }^{1}$. Conjointement, l'intention des couples demandeurs de parler à leur enfant du recours aux gamètes d'un tiers y apparaît majoritaire (65 \%)

\footnotetext{
* Auteur correspondant.

Adresse e-mail : marjolaine.doumergue@univ-lyon2.fr (M. Doumergue).

${ }^{1}$ E.g., ces 15 dernières années, 4 pays européens ont mis fin à l'anonymat du donneur, rejoignant 4 autres l'ayant fait à partir de 1985 .
}

[1,2], alors que dans les études internationales, quel que soit le statut du donneur, le secret à l'enfant prévaut (En Suède, où l'anonymat était proscrit, $15 \%$ des parents informaient leurs enfants [3]. Une étude européenne [4] (pays avec anonymat) stipulait que 8,6\% des parents avaient parlé à leurs enfants de 11 ans - contre 69,9 \% ayant décidé de ne rien leur dire). Ce contraste rend particulièrement saillante la rareté des données françaises sur le devenir des récits de conception dans les familles constituées suite à une procréation avec don de spermatozoïdes (DSP). Grâce à un partenariat de recherche avec la Fédération française des Cecos, nous avons rencontré des parents aux temps et 
lieux institutionnels de leur seconde démarche. Ce contexte précis conditionne, mais aussi rend possible le recueil de données auprès de cette population sensible. Nous nous intéressons à la manière dont les parents pensent, expérimentent, pratiquent leurs récits de parenté. Les récits de conception renseignent ainsi sur l'expérience des parents de ce mode de parenté et leur perception du donneur. Notre approche consiste à les appréhender à la fois comme narrations, enjeu pratique et processus stratégique $[5,6]$.

\section{Méthodologie}

Entre 2008 et 2012, 929 requérants d'un DSP (373 couples et 183 personnes), présents au sein de 20 Cecos, ont été interrogés individuellement par questionnaire. Parmi les parents, 23,1\% ( $n=216$ ) étaient d'au moins un enfant né par DSP (âge moyen $=31$ mois \pm 25 mois). Parmi les répondants, 74,8 \% étaient recrutés par les médecins ou psychologues lors des consultations ayant lieu pendant la période d'attente, 25,2 \% pendant leurs traitements. L'échantillon est similaire par ces caractéristiques à la file active des requérants d'un don de spermatozoïdes aux Cecos [7]. Nous présenterons les résultats relatifs à leur attitude quant à l'encadrement législatif, à leur perception du donneur et à leur décision de partage à l'enfant des conditions de sa conception. Les différences de réponses des parents et des primo-requérants ont été analysées par $\mathrm{X}^{2}$ et Anovas à un facteur.

En plus, un échantillon de 37 parents ( 17 couples et 3 femmes) a été recruté au sein de 8 Cecos, lors de leur démarche pour un deuxième ou troisième enfant, en vue d'entretiens individuels semi-directifs. Au sein de ces 20 familles, 22 enfants étaient nés suite à une procréation avec donneur. Ils étaient âgés de 8 mois à 6,5 ans (âge moyen 23 mois $\pm 19,9$ mois ; 3 enfants avaient plus de 4 ans). Nous présenterons ici les résultats qualitatifs centrés sur les récits de conceptions proposés ou prévus par les parents.

\section{Résultats}

Les résultats quantitatifs sont caractérisés par une grande homogénéité entre sous-groupes, les réponses des parents et des primo-requérants ne différant que localement. Les parents ont adhéré, plus encore $(p<, 05)$ que les primo-requérants, aux principes législatifs régissant le DSP. À 95,3\%, ils ont plébiscité un DSP " anonyme et gratuit » (vs 92,3\%). Pour tous, la perception du donneur anonyme est ambivalente. Il est dépersonnalisé (55,4\%), mais suscite un intérêt exprimé par un sentiment de gratitude ( $59 \%$ ) ou la crainte d'éventuels problèmes médicaux de l'enfant $(77,8)$. Enfin, les parents, comme les primo-requérants, adhèrent majoritairement $(68,8$ \%) à l'idée de parler à l'enfant de sa conception avec donneur.

\subsection{Objectivation du donneur dans les récits : la métaphore de la graine}

"Parce qu'on veut lui dire " nous informaient spontanément quasiment tous les parents lors des entretiens, tandis que 2/ 3 d'entre eux se reprenaient d'emblée pour admettre en avoir déjà parlé à l'enfant. Il s'agissait de trouver les " bons mots ", avant que le « concret des vrais termes " puisse être énoncé :

- « Parler d'ovules et de spermatozoïdes d'emblée, avec l'insémination, à 2 ans, je ne pense pas. Il y aura une évolution dans l'affinement de ma réponse. Mais je pense que dès qu'elle sera prête à entendre et à comprendre, en fonction de son âge, elle aura la méthode claire et nette " (mère, enfant 11 mois) ;

- "À 3 ans, on ne va pas lui dire qu'on a galéré 5 ans pour l'avoir " (père, enfant 8 mois) ;
- "Il faut commencer à en parler de la façon la plus poétique possible et puis, après, rentrer un peu plus dans les détails au fur et à mesure de l'avancement de l'âge de l'enfant " (père, enfant 10 mois).

L'enjeu réside dans la manière de faire figurer le donneur au sein du récit. Les versions confiées s'accordent quasi unanimement sur la " métaphore de la graine " qui recouvre néanmoins une perception différenciée du donneur et de son intervention :

1 « Il y avait la graine de maman, mais chez papa, les graines, elles étaient cassées, donc on était obligé de demander une graine au docteur " (mère, enfant 2 ans) ;

2 " On s'était rendu compte que papa, il n'avait pas de petites graines à planter. Alors, l'hôpital, c'est un peu la jardinerie, on va chercher la petite graine " (mère, enfant 1 an);

3 " Il manquait des petites graines à papa, on a été chercher des graines ailleurs " (père, enfant 10 mois) ;

4 " Papa est allé chercher une petite graine parce que je n'en avais pas, il y a un gentil papa qui en avait beaucoup, et donc il en a donné une " (père, 2 enfants de 2 ans) ;

5 " Papa c'est ton papa, et puis il y a un autre monsieur qui a le même $A D N$ que papa qui a donné ses graines pour que tu sois là. Parce que papa, il n'a pas de graines " (père, enfant 18 mois).

En effet, certains (ex. 1 et 2) éludent son rôle en ne mettant l'accent que sur l'aide médicalisée et l'institution. L'extrait 3 est emblématique de récits mentionnant des graines " autres " venues d'un allusif " ailleurs ". D'autres encore (4 et 5) soulignent l'intervention d'un donneur, respectivement qualifié comme un père ou comme génétiquement proche du père, sans faire état explicitement de la médiation institutionnelle. Notons également que la métaphore botanique est parfois filée et permet ensuite de dissocier étapes et rôles (fournir la graine, la planter, faire grandir la plante, etc.). Il ne s'agit pas ici de pointer l'incomplétude de brefs instantanés, puisque les parents pensent qu'ils évoluent et évolueront.

Ce cheminement se laisse particulièrement appréhender par l'impression pour $2 / 3^{2}$ des parents d'un récit déjà-là mais toujours à venir :

- " [On le dira quand ils demanderont comment on fait les bébés]. Mais d'ailleurs, je leur dis déjà qu'ils viennent de graines magiques. Ça, je leur ai raconté cent-mille fois déjà " (mère, 2 enfants de 2 ans) ;

- "J'ai beaucoup d'appréhension sur ça et. . . parce qu'on va lui dire, hein. Enfin, on lui dit déjà, mais des appréhensions du style « Ben tu n'es pas mon père, je peux faire ce que je veux " " (père, enfant 11 mois).

Amenés alors à parler du récit précoce (parfois dès la maternité), les parents témoignent d'un ensemble d'objectifs : globalement, " qu'il n'y ait jamais eu de tabou » avec l'enfant, puis qu'il s'imprègne de l'histoire, se familiarise avec elle et se l'approprie : " c'est son histoire maintenant ". En somme, le pari consiste en une familiarisation progressive de l'enfant avec une histoire qui deviendra autant " naturelle " que " normale ".

\subsection{Mise en échec des récits : la crainte de la recherche des origines}

Interrogés sur l'avenir du récit de conception avec l'avancée en âge des enfants, les parents signifiaient qu'ils l'adapteraient en fonction des questions ultérieures de l'enfant. Espérées, ces questions sont également redoutées. Les parents s'attendent tous

\footnotetext{
${ }^{2}$ Le tiers restant souhaitait amorcer l'histoire avec les questions de l'enfant ou lors d'une seconde grossesse.
} 
à ce qu'à l'adolescence, elles puissent se muer en remise en question du père. Dans la plupart de leurs discours, cette crainte est mêlée à celle d'un intérêt porté par l'adolescent à la " recherche des origines » et forcément au donneur. Ainsi, les figures du donneur et du père apparaissaient exclusives, en vases communicants, et non cumulatives :

- " Ça me ferait un peu mal qu'A. [l'enfant] mette un point d'honneur à retrouver l'identité du donneur. Ça me montrerait qu'il nie, en quelque sorte, ma paternité alors que le projet, c'est pas celui du donneur " (père, enfant 10 mois) ;

- " [Sans anonymat] j'aurais été beaucoup plus... pas distant... mais méfiant, justement, en appréhension avec le fait que mon enfant veuille connaître ses origines. Et jusqu'où ils veulent aller... il veut aller et. . . Qu'est-ce que je peux. . . Ce n'est pas ma possession, ce n'est pas ce que je veux dire. Mais c'est quelqu'un qu'on élève. Et de se dire qu'on le perd à la fin parce qu'il préfère voir quel est son vrai père biologique, ce qu'il aurait pu lui donner en plus que moi je ne peux pas donner. . . " (père, enfant 11 mois).

Ainsi, la recherche du donneur équivaudrait pour beaucoup de parents interrogés à l'échec de l'intégration par l'enfant des enjeux de son récit de conception, par une association réalisée entre don et paternité. Cette association est dénotée également dans les discours par une scène, maintes fois imaginée, d'un jeune majeur sonnant à la porte du donneur et s'adressant à lui en des termes paternels. Pour une majorité de parents, l'anonymat est ainsi d'abord la garantie de l'absence de toute interaction possible entre donneur et enfants qui serait empreinte d'une idée de filiation. Des nuances étaient apportées par certains parents (moins d'1/3) évoquant le choix ou l'intérêt des enfants à pouvoir identifier le donneur, envisageant d'aider les leurs dans leurs recherches éventuelles ou partageant leurs interrogations pressenties. Par ailleurs dans les entretiens, l'anonymat garantissait, bien plus fréquemment que la reconnaissance légale en paternité, l'absence de droits du donneur sur la famille. Cependant, aucun interviewé n'a fait de lien entre l'anonymat du donneur et sa décision d'en parler.

\section{Discussion}

Nos données sur les décisions parentales du récit à l'enfant de sa conception avec donneur supportent l'idée d'une relative indépendance entre celles-ci et le statut du donneur (anonyme en France) $[2,8]$. En effet, les intentions majoritaires sont similaires à celles constatées récemment en Finlande où l'anonymat prévalait [9] et en Suède où le donneur est identifiable à la majorité de l'enfant, depuis 1985 [10]. Ce, alors que les répondants français manifestent une adhésion massive à l'anonymat, cadre premier au sein duquel s'est déployée leur réflexion sur le DSP. Cette " appropriation culturelle " ne minore en rien sa fonction : l'anonymat a un rôle signifiant de dissociation de la paternité, et instituant, en ce qu'il supplée fréquemment, dans les représentations sociales de la parenté [11], la reconnaissance en paternité pour instaurer le père comme père. Aussi une interaction enfant/ donneur qui ne soit pas inscrite dans un paradigme filial apparaîtelle pour beaucoup irreprésentable.

La perception du donneur, déjà ambivalente chez les couples demandeurs, n'est pas complètement pacifiée chez les parents [12]. Cette perception se déploie le long d'une tension entre « être et faire ", ou " substance et relation ", ou " bien et lien ". Au sein des différentes narrations, la figure du donneur navigue entre la réification à une exo-graine, l'idée d'un double du père, un homme qualifié par sa générosité, voire une personne connue. Reste que les " bons mots " se trouvent avec la métaphore de la graine. Ses fonctions sont plurielles : intelligible, désexualisée, " naturelle", elle évoque l'obstacle rencontré par les couples, concrétise et symbolise l'aide reçue. Ainsi, l'objectivation du donneur sous ce motif rend saillant le bien, la chose donnée ; la relation étant l'apanage du père. Pour autant, le donneur n'est absent ni des récits, ni de ses intentions sous-jacentes. La négation de l'acte de don est inenvisageable, et son oubli, pourtant souhaité, jugé nécessaire, est impossible. Nous retrouvons ainsi inhérentes à la figure du donneur les tensions croisées entre constructions biogénétiques et socio-environnementales de la famille, déjà décrites [12,13], et celles liées au don. Le don, relation réciproque d'échange de choses, est appréhendé via l'anonymat comme une " relation de non-relation ", où se négocie "l'active présence de l'absence " du donneur [14].

La métaphore de la graine traduit cette négociation, contraction saisissante entre donneur et bien donné, déjà-là et à venir. La stratégie d'un récit-processus le fait également, l'histoire et ses détails grandissant avec l'enfant et sa compréhension [5,6]. Le projet représentationnel exprimé par les parents s'y appuie et vise en une normalisation de ce mode de conception. La familiarisation de l'enfant avec celui-ci est prévue comme contexte premier afin que la personne du donneur ne lui soit qu'une vérité dernière [12]. L'information quant à celle-ci est fournie en même temps que sa signification : sa non-participation de plain-pied au schéma familial.

\section{Déclaration d'intérêts}

Les auteurs déclarent ne pas avoir de conflits d'intérêts en relation avec cet article.

Financements : Agence de la biomédecine (PEGH 2010); région Rhône-Alpes (CIBLE 2010).

Partenaire scientifique : Fédération française des Cecos.

\section{Références}

[1] Kalampalikis N, Doumergue M. Le don et son récit. Paradoxes bioéthiques et enjeux psychosociaux du don de sperme. Région Rhône-Alpes, GRePS Lyon 2: Fédération française des Cecos, Agence de la biomédecine; 2013.

[2] Jouannet P, Kunstmann JM, Juillard JC, Bresson JL. La Fédération française des Cecos. La majorité des couples procréant par don de sperme envisagent d'informer l'enfant de son mode de conception, mais la plupart souhaitent le maintien de l'anonymat du donneur. Andrologie 2010;20:29-36.

[3] Gottlieb C, Lalos O, Lindblad F. Disclosure of donor insemination to the child: the impact of Swedish legislation on couples' attitudes. Hum Reprod 2000:15:2052-6.

[4] Golombok S, Brewaeys A, Giavazzi MT, Guerra D, Mc-Callum F, Rust J. The European study of assisted reproduction families: the transition to adolescence. Hum Reprod 2002;17:830-40.

[5] McDougall K, Becker G, Scheib JE, Nachtigall RD. Strategies for disclosure: how parents approach telling their children that they were conceived with donor gametes. Fertil Steril 2007;87:524-33.

[6] Readings J, Blake L, Casey P, Jadva V, Golombok S. Secrecy, disclosure and everything in-between: decisions of parents of children conceived by donor insemination, egg donation and surrogacy. Reprod BioMed Online 2011:22:485-95.

[7] Hennebicq S, Julliard J-C, Le Lannou D. Données descriptives de l'activité d'AMP avec don de spermatozoïdes au sein des Cecos de 1973 à 2006 en France. Andrologie 2010;1:3-10.

[8] Indekeu A, Dierickx K, Schotsmans P, Daniels K, Rober P, D'Hooghe T. Factors contributing to parental decision-making in disclosing donor conception: a systematic review. Hum Reprod Update 2013;6:714-33.

[9] Sälevaara M, Suikkari A, Söderström-Anttila V. Attitudes and disclosure decisions of Finnish parents with children conceived using donor sperm. Hum Reprod 2013;10:2746-54.

[10] Isaksson S, Sydsjö G, Skoog Svanberg A, Lampic C. Disclosure behavior and intentions among 111 couples following treatment with oocytes or sperm from identity-release donors: follow-up at offspring age 1-4 years. Hum Reprod 2012;27:2998-3007.

[11] Moscovici S. Le scandale de la pensée sociale. Paris: Ehess: 2013.

[12] Indekeu A, D'Hooghe T, Daniels K, Dierickx K, Rober P. When “sperm” becomes "donor": transition in parents' views of the sperm donor. Hum Fertil 2014:0:1-9.

[13] Grace V, Daniels K, Gillett W. The donor, the father, and the imaginary constitution of the family: parents' constructions in the case of donor insemination. Soc Sci Med 2008;66:301-14.

[14] Konrad M. Nameless relations. Oxford: Berghahn Books; 2005. 